\title{
USPI 3 serves as a tumor suppressor via the PTEN/AKT pathway in oral squamous cell carcinoma
}

This article was published in the following Dove Medical Press journal: Cancer Management and Research

\author{
Zhi Qu' \\ Ran Zhang ${ }^{2}$ \\ Meng Su${ }^{2}$ \\ Weixian Liu' \\ 'Department of Oral and Maxillofacial \\ Surgery, Shengjing Hospital, China \\ Medical University, Shenyang, China; \\ ${ }^{2}$ Department of Prosthodontics, \\ Second Affiliated Hospital of Jinzhou \\ Medical University, Jinzhou, China
}

Background: Recent studies have shown that USP13 a deubiquitinase, serves as an important regulator of tumorigenesis. However, the biological role of USP13 in oral squamous cell carcinoma (OSCC) remains enigmatic.

Materials and methods: We examined USP13 expression in OSCC and adjacent normal tissues by immunohistochemical staining. The biological functions of USP13 in OSCC cells and the possible underlying mechanisms were investigated.

Results: In this study, we showed that USP13 expression was frequently reduced in human OSCC specimens and that the reduction was correlated with the clinical stage. Functional studies demonstrated that overexpression of USP13 suppressed OSCC cell proliferation, glucose uptake and lactate production in vitro and inhibited tumor growth in vivo. Furthermore, USP13 overexpression induced phosphatase and tensin homolog deleted on chromosome 10 (PTEN) expression and repressed the activation of AKT as well as the expression of the downstream effectors glucose transporter-1 (GLUT1) and hexokinase-2 (HK2). Overexpression of PTEN reversed the USP13-knockdown-induced glucose uptake, lactate production, AKT activation, and expression of GLUT1 and HK2.

Conclusion: Our findings suggest that USP13 serves as a tumor suppressor by regulating the PTEN/AKT signaling pathway in OSCC cells, improving our understanding of OSCC progression and providing a clue for the development of a novel cancer therapy.

Keywords: OSCC, USP13, PTEN, glycolysis

\section{Introduction}

Oral squamous cell carcinoma (OSCC) accounts for $>40 \%$ of head and neck cancers and is the sixth most common cancer in the world. ${ }^{1}$ Despite the progress in diagnosis and treatment, the 5-year survival rate of OSCC is still far from satisfactory. ${ }^{2}$ Therefore, a better understanding of the pathophysiological mechanisms and molecular events involved in the development of OSCC is urgently needed to promote prognostic stratification and clinical management in patients with OSCC.

The metabolism of malignant cells is different from that of normally differentiated cells. Oxidative phosphorylation in mitochondria provides energy to the normally differentiated cells, while aerobic glycolysis provides energy to cancer cells growing in uncontrolled conditions. ${ }^{3,4}$ This phenomenon is called the Warburg effect and is critical for maintaining the high proliferation rate of cancer cells. ${ }^{3} \mathrm{~A}$ high rate of glucose uptake and lactate production is the main characteristic of the Warburg effect. ${ }^{5}$
Correspondence: Weixian Liu Department of Oral and Maxillofacial Surgery, Shengjing Hospital, China Medical University, No.36 Sanhao Street, Heping District, Shenyang, China Email liuweixian6666@163.com 
Phosphatase and tensin homolog deleted on chromosome 10 (PTEN), a tumor suppressor gene, can suppress AKT activation by antagonizing the effect of phosphatidylinositol-3-kinase (PI3K). ${ }^{6}$ PTEN expression ${ }^{7,8}$ is downregulated in OSCC and associated with disease stage and lymph node metastasis, while activated AKT is an independent prognostic factor for OSCC. ${ }^{910}$ Activated AKT has been reported to stimulate cell proliferation and glycolysis in cancer cells. ${ }^{11-13}$

The deubiquitinase USP13 is a member of the USP subclass of the deubiquitinating enzyme superfamily. ${ }^{14}$ USP13 can remove ubiquitin chains from its substrates to inhibit protein degradation. ${ }^{15}$ It has been reported that USP13 is involved in cell cycle regulation, endoplasmic reticulum-associated degradation, autophagy, and innate antiviral immunity by regulating its substrates $\operatorname{Skp} 2,{ }^{16}$ Ub14A, ${ }^{17}$ Beclin- $1,{ }^{18}$ and STING. ${ }^{19}$ Recently, controversial functions of USP13 in tumorigenesis have been reported. USP13 promotes melanoma cell invasion by stabilizing the microphthalmia-associated transcription factor. ${ }^{20}$ In addition, USP13 is aberrantly overexpressed in ovarian cancers, and USP13 drives ovarian cancer metabolism by deubiquitinating ATP citrate lyase and oxoglutarate dehydrogenase. ${ }^{21}$ USP13 disruption inhibited the proliferation of glioma stem cells by promoting the ubiquitination and degradation of c-Myc. ${ }^{22}$ However, USP13 also plays a tumor-suppressive role in breast cancer by stabilizing PTEN. ${ }^{23}$ USP13 is identified as a target of miRNA-135b ${ }^{24}$ in colorectal cancer cells, which promotes colorectal cancer cell proliferation and glycolysis.

In the present study, we observed downregulation of USP13 in OSCC tissues, which was strongly associated with clinical stage. USP13 overexpression inhibited the proliferation of and glycolysis in OSCC cells in vitro and suppressed tumorigenesis in nude mice. Moreover, the PTEN/ AKT pathway may mediate the inhibitory effects of USP 13 on glycolysis.

\section{Materials and methods}

\section{Tissue samples and patient information}

The paraffin sections, including 50 cases of human OSCC and 10 of adjacent tissues were purchased from Outdo Biotechnology (Shanghai, China). The mean age of the patients was $58.5 \pm 10.4$ years, and $56.0 \%$ of patients were male $(\mathrm{n}=28)$. Thirty-eight patients had stage I or II cancer, and 12 had stage III or IV cancer. This study was approved by the Ethical Committee of Shengjing Hospital, China Medical University.

\section{Immunohistochemical analysis}

The paraffinized sections were deparaffinized with xylene and rehydrated with a series of ethanol solutions as previously described. ${ }^{23}$ To expose antigen epitopes, the sections were heated in a pressure cooker for 10 minutes in $0.1 \mathrm{M}$ citric acid buffer (pH 6.0). After cooling, endogenous peroxidase activity was blocked by immersing the sections in $0.3 \%$ hydrogen peroxide for 15 minutes. The sections were incubated with rabbit anti-USP13 (16840-1-AP, ProteinTech, Chicago, IL, USA) overnight at $4^{\circ} \mathrm{C}$ and then with horseradish peroxidaseconjugated goat anti-rabbit secondary antibodies. The signals were visualized using a 3,3'-diaminobenzidine (DAB) kit (Long Island, Shanghai, China).

\section{Cell culture}

CAL27, HSC4, SCC15, and 293 T cells were obtained from the Cell Bank of Chinese Academy of Sciences (Shanghai, China). All the cell lines were cultured in high glucose DMEM (HyClone, Logan, UT, USA) containing 10\% FBS (Invitrogen, Carlsbad, CA, USA) and penicillin/streptomycin (Solarbio, Beijing, China) at $37^{\circ} \mathrm{C}$ in a moist atmosphere with $5 \% \mathrm{CO}_{2}$.

\section{Lentivirus-mediated overexpression}

The coding sequence of USP13 or PTEN was amplified with the following primers: USP13 (NM_003940.2), forward 5'-CGGAATTCATGCAGCGCCGGG-3' and reverse 5'-CGGGATCCTTAGCTTGGTATCCTGCGG-3'; and PTEN (CR450306.1), forward 5'-CGGAATTCATGACAGCCATCATCAAAGAG-3' and reverse 5'-CGGGATCCCGATCTCTTTGATGATGGCTG-3'. After digestion, the DNA fragments were cloned into a linearized pLVX-puro expression vector (Clontech, Palo Alto, CA, USA) and the constructs were confirmed by DNA sequencing. For virus production, the constructs together with the packaging plasmids psPAX2 and pMD2G (Addgene, Cambridge, MA, USA) were transfected into $293 \mathrm{~T}$ cells with lipofectamine 2000 (Invitrogen). Lentiviruses were collected from the culture medium 48-72 hours after transfection.

\section{Lentivirus-mediated RNAi}

RNAi oligos targeting USP13 were cloned into linearized pLKO. 1 plasmids (Addgene) and confirmed by DNA sequencing. The interference sites are as follows: shUSP13\#1 (position 761-779), 5'-GCTCTGTCCTGTGTGGAAA-3'; shUSP13\#2 (position 851-869), 5'-CCGT- 
GAAACTGGGAACCAT-3'; and shUSP13\#3 (position 1052-1070), 5'-GCACGAAACTGAAGCCAAT-3'. The lentivirus was packaged as described previously.

\section{Protein extraction and Western blot analysis}

Protein was extracted using RIPA buffer (Jrdun Biotech., Shanghai, China) containing protease and phosphatase inhibitors and then subjected to $10 \%$ SDS-PAGE. After transferring the protein to nitrocellulose membranes (Millipore, Billerica, MA, USA), nonspecific binding was blocked by $5 \%$ nonfat milk in Tris-buffered saline containing $0.1 \%$ Tween-20 (TBST). Subsequently, the membranes were probed with the following primary antibodies overnight at $4{ }^{\circ} \mathrm{C}$ : USP13 (Ab109264, Abcam, Cambridge, MA, USA), glucose transporter-1 (GLUT1) (Ab115730, Abcam), hexokinase-2 (HK2) (Ab104836, Abcam), PTEN (\#9552, Cell Signaling Technology, Danvers, MA, USA), p-AKT (\#9271, Cell Signaling Technology), AKT (\#9272, Cell Signaling Technology), and glyceraldehyde3-phosphate dehydrogenase (\#5174, Cell Signaling Technology). Following three washes with TBST, horseradish peroxidase conjugated secondary antibody was applied at room temperature for 1 hour. The signals were developed using the enhanced chemiluminescent substrate (Bio-Rad, Richmond, CA, USA).

\section{Cell proliferation assay}

A Cell Counting Kit-8 (CCK-8, CP002, SAB biotech., College Park, MD, USA) assay was carried out following the manufacturer's protocol to assess cell proliferation. In brief, the cells at a logarithmic growth phase were collected and adjusted to $3 \times 10^{4}$ cells $/ \mathrm{mL}$. Cell suspension was added to 96-well plates $(100 \mu \mathrm{L} /$ well $)$ and cultured overnight. CAL27 and HSC 4 cells were transduced with USP13 overexpressing lentivirus (USP13OE) or a control virus (Vector). Cells not undergoing any treatment were used as the control. After culturing for the indicated time periods, $\mathrm{CCK}-8$ solution was added to each well and incubated at $37^{\circ} \mathrm{C}$ for 1 hour. OD was measured at a wavelength of $450 \mathrm{~nm}$ on a microplate absorbance reader (Bio-Rad).

\section{Measurement of 2-(N-[7-nitrobenz- 2-oxa-I,3-diazol-4-yl]amino)-2- deoxyglucose (2-NBDG) uptake}

The OSCC cell lines were plated in 6 -well plates $\left(5 \times 10^{5}\right.$ cells/ well) and treated as indicated in the figure legends for 48 hours. Subsequently, the cells were incubated with glucosefree Krebs-Ringer buffer (KRB) at $37^{\circ} \mathrm{C}$ for 15 minutes and then with $100 \mu \mathrm{M}$ 2-NBDG (Cayman, Ann Arbor, MI, USA) in glucose-free medium (Gibco, Grand Island, NY, USA) at $37^{\circ} \mathrm{C}$ for 45 minutes. After washing with $\mathrm{KRB}$ buffer three times, the cells were harvested and fluorescent density was measured using flow cytometry (BD Biosciences, Franklin Lakes, NJ, USA). The values of the control group were set to 1 .

\section{Lactate production}

The OSCC cells in the 6-well plates were treated as indicated in the figure legends. After 48 hours of culture, the medium was collected to measure lactate production with a lactic acid (LD) detection kit (Nanjing Jiancheng Bioengineering Institute, Nanjing, China) as per the manufacturer's protocol. The values of control group were set to $100 \%$.

\section{Tumor growth}

The animal experiment was performed in accordance with the guidelines for the Care and Use of Laboratory Animals and approved by Ethical Committee of Shengjing Hospital, China Medical University. Female athymic nude mice from the Shanghai Laboratory Animal Center (Shanghai, China) were maintained in specific-pathogen-free conditions. CAL27 cells expressing USP13 or a vector were collected and adjusted to $5 \times 10^{7} / \mathrm{mL}$ in FBS-free medium. The nude mice were randomly divided into two groups ( $n=6$ per group) and injected subcutaneously with $100 \mu \mathrm{L}$ of cell suspension. After the xenograft formed, the length and width of the xenograft were monitored every 3 days, and tumor volume was calculated using the following equation: volume $=1 / 2 \times$ length $\times$ width $^{2}$ as previously described. ${ }^{25}$ On day 33 , the mice were sacrificed. The xenografts were resected and weighed, and partial tissues were processed for Western blotting analysis.

\section{Statistical analysis}

GraphPad Prism (GraphPad Software, San Diego, CA, USA) was used for all statistical tests. Fisher's exact test was performed to analyze the correlation of USP13 expression with the clinical features of OSCC patients. Statistical analyses of in vitro experiments were conducted by ANOVA. Student's $t$-test was used to compare the significant differences in the nude mice experiment. $P<0.05$ was considered statistically significant. 


\section{Results}

\section{Decreased expression of USPI 3 in OSCC tissues}

To discover USP13 expression in OSCC tissues, we performed immunohistochemistry staining on 50 samples of human OSCC and 10 of adjacent tissues. Low expression of USP13 (with $>25 \%$ of tumor cells positively stained) was detected in 31 cases of OSCC, whereas high expression of USP13 (with no $>25 \%$ of tumor cells positively stained) was observed in the other 19 cases (Figure 1). Fisher's exact test indicated that USP13 expression was strongly correlated with clinical stage $(P<0.05)$ but not with age, gender, or pathologic differentiation (Table 1).

\section{USPI 3 overexpression repressed cell proliferation and the Warburg effect in OSCC cells}

Given that USP13 expression was associated with clinical stage of OSCC, we supposed that USP13 expression may
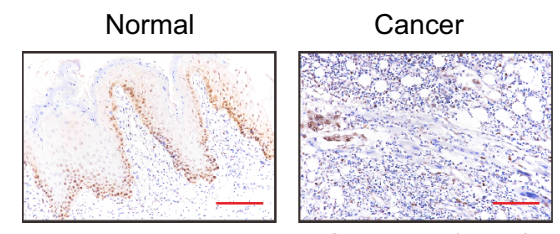

USP13 low $(n=31)$

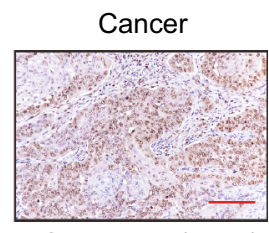

USP13 high $(n=19)$
Figure I Decreased expression of USPI 3 in OSCC.

Notes: USPI 3 protein expression was determined by immunohistochemistry staining in OSCC and normal tissues. Low and high expression of USPI3 was detected in $3 \mathrm{I}$ and 19 OSCC patients, respectively. The scale bar: $100 \mu \mathrm{m}$.

Abbreviation: OSCC, oral squamous cell carcinoma.

Table I Correlation of USPI 3 expression in OSCC tissues with different clinicopathological features $(n=50)$

\begin{tabular}{|c|c|c|c|}
\hline \multirow[t]{2}{*}{ Characteristic } & \multicolumn{2}{|l|}{ USPI3 } & \multirow[t]{2}{*}{$P$-value } \\
\hline & $\begin{array}{l}\text { High } \\
(n=19)\end{array}$ & $\begin{array}{l}\text { Low } \\
(n=31)\end{array}$ & \\
\hline \multicolumn{3}{|l|}{ Gender } & \multirow[t]{3}{*}{0.7742} \\
\hline Male & 10 & 18 & \\
\hline Female & 9 & 13 & \\
\hline \multicolumn{3}{|l|}{ Age (years) } & \multirow[t]{3}{*}{$0.556 I$} \\
\hline$\geq 60$ & 8 & 10 & \\
\hline$<60$ & 11 & 21 & \\
\hline \multicolumn{3}{|l|}{ Clinical stage } & \multirow[t]{3}{*}{$0.0182 *$} \\
\hline $\mathrm{I} / \mathrm{II}$ & 18 & 20 & \\
\hline III/IV & $\mathrm{I}$ & 11 & \\
\hline \multicolumn{3}{|l|}{ Pathologic differentiation } & \multirow[t]{3}{*}{0.4524} \\
\hline Well/moderate & 17 & 24 & \\
\hline Undifferentiated & 2 & 7 & \\
\hline
\end{tabular}

Note: The Fisher's exact test was performed. ${ }^{*} P<0.05$.

Abbreviation: OSCC, oral squamous cell carcinoma. affect OSCC cell proliferation. To test this hypothesis, we overexpressed USP13 by a lentivirus-mediated system in CAL27 and HSC4 cells, which had relatively low levels of USP13 (Figure S1). The lentivirus overexpressing USP13 effectively upregulated the expression of USP 13 compared with the control lentivirus (vector) and the control cells that did not undergo any treatment (Figure 2A). We found significant decreases in cell proliferation of CAL27 and HSC4 cells (Figure 2B, $P<0.01$ ) with USP13 overexpression (USP13OE).

The Warburg effect supplies energy to tumor cells to maintain a high proliferation rate. ${ }^{3}$ Thus, we subsequently detected the alterations in glucose uptake and lactate production. Significant decreases in 2-NBDG uptake (Figure 2C, $P<0.001$ ) and lactate production (Figure 2D, $P<0.001$ ) were observed in CAL27 and HSC4 cells with USP13 overexpression (USP13OE).

Real-time PCR was then performed to detect the expression of glycolysis-related genes, including PKM2, HIF1A, HK2, GLUT1, GLUT4, LDHA, and c-Myc (Supplemental materials, Figure S2). The mRNA levels of GLUT1 and HK2 changed the most with USP13 overexpression. As shown in Figure 2E, the Western blotting analysis also illustrated that USP13 overexpression led to an obvious reduction in the protein levels of GLUT1 and HK2. The PTEN/AKT pathway is known to play a critical role in glycolysis. ${ }^{6}$ The PTEN protein level was increased and the phosphorylation of AKT (p-AKT) was reduced by the overexpression of USP13 (Figure 2F). These data suggested that USP13 overexpression inhibited glycolysis in OSCC cells.

\section{The PTEN/AKT pathway was involved in the biological functions of USPI 3 in OSCC cells}

To corroborate the role of the PTEN/AKT pathway in the biological functions of USP13 in OSCC cells, USP13 expression was downregulated and that of PTEN was upregulated in SCC15 cells, which displayed a relatively higher expression of USP13. Figure 3A demonstrates that all lentivirusmediated RNAi against USP13 (shUSP13\#1, shUSP13\#2, and shUSP13\#3) effectively downregulated the expression of USP13 compared with the control lentivirus (shNC), and shUSP13\#2, the most efficient lentivirus-mediated RNAi, was used in the subsequent experiments. Figure 3B shows that lentivirus overexpressing PTEN (PTENOE) effectively upregulated the expression of PTENOE.

Notably, overexpression of PTEN partially abolished shUSP13\#2-stimulated glucose consumption (Figure 3C), lactate production (Figure 3D), protein expression of GLUT1 
A

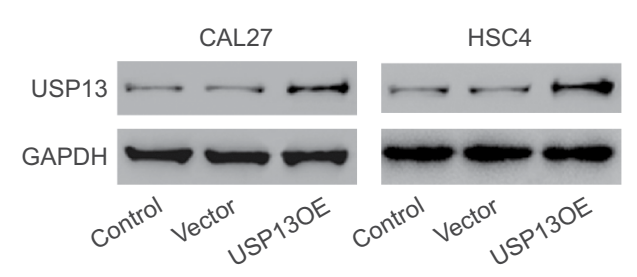

C

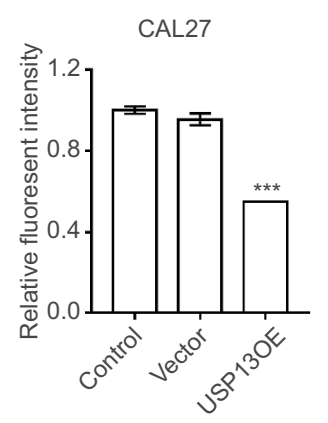

E

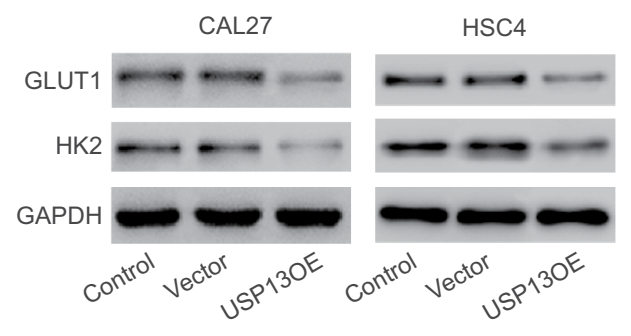

B
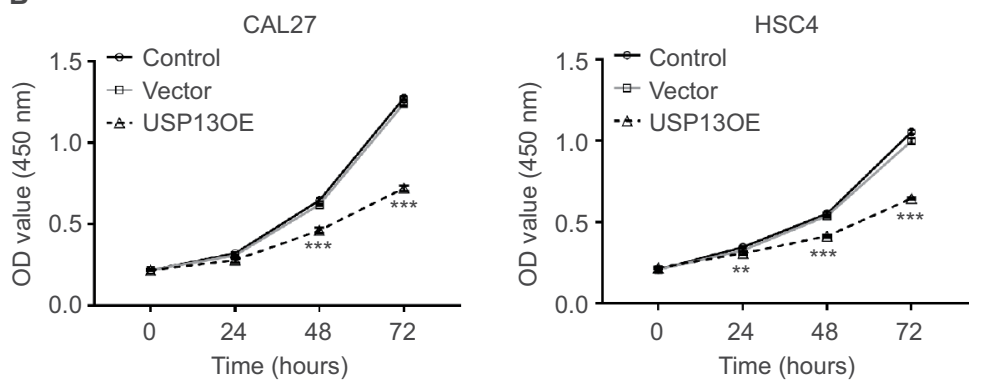

D
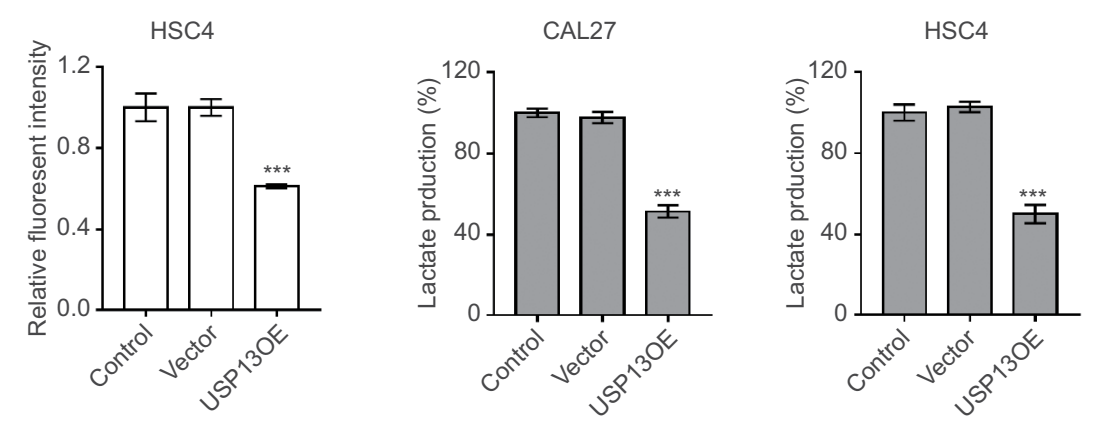

$\mathbf{F}$

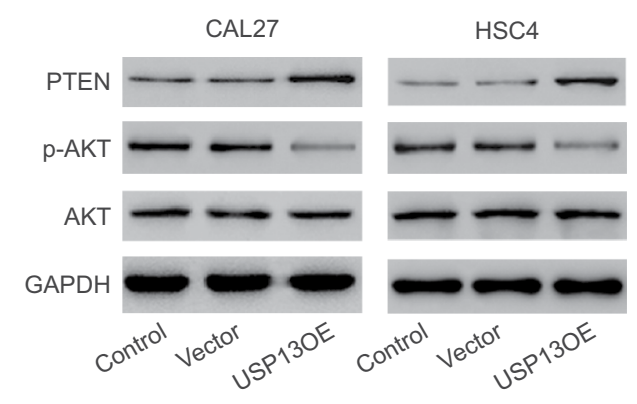

Figure 2 USPI 3 overexpression suppressed OSCC cell proliferation and the Warburg effect.

Notes: (A) CAL27 and HSC4 cells were transduced with a USPI 3-overexpressing lentivirus (USPI3OE) and a control vector virus. Cells that did not undergo any treatment were used as a control. Western blot was performed to detect USPI3 expression 48 hours post treatment. Representative blots from three independent experiments are shown. (B) A CCK-8 assay was performed to assess cell proliferation ( $n=3$ biological replicates) in CAL27 and HSC4 cells with USPI3OE and the control vector. (C) 2-NBDG uptake was measured ( $n=3$ biological replicates) 48 hours post treatment. Fluorescent density was measured using flow cytometry and the values for the control group were set at I. (D) Lactate production was measured ( $n=3$ biological replicates) and the values for the control group were set at $100 \%$. (E, F) Western blot was performed to detect the related proteins. Representative blots from three independent experiments are shown. $* * P<0.0 \mathrm{I}$, $* * * P<0.00 \mathrm{I}$ vs the control and shNC.

Abbreviations: CCK-8, Cell Counting Kit-8; GAPDH, glyceraldehyde-3-phosphate dehydrogenase; GLUTI, glucose transporter-I; HK2, hexokinase-2; 2-NBDG, 2-(N-[7nitrobenz-2-oxa-1,3-diazol-4-yl]amino)-2-deoxyglucose; shNC, control shRNA; OSCC, oral squamous cell carcinoma; PTEN, phosphatase and tensin homolog deleted on chromosome 10.

and HK2 (Figure 3E), and phosphorylation of AKT (Figure $3 \mathrm{~F}$ ) in SCC15 cells. These data suggested that the PTEN/AKT pathway mediated the effects of USP13 on the glycolysis in OSCC cells.

\section{Effect of USPI 3 overexpression on OSCC in vivo}

To determine the direct function of USP13 on OSCC growth, we evaluated the growth ability of CAL27 cells overexpress- ing USP13 in nude mice. The tumor growth curve in Figure 4A shows that the volumes of tumors formed from cells overexpressing USP13 (USP13OE) were evidently smaller than those formed from cells with the vector from day 12 to day 33 after implantation. On day 33, the weight of the USP13OE tumors was significantly lighter than that of the vector (Figure 4B, $P<0.001$ ). The Western blotting analysis in Figure 4C, D demonstrated the elevated expression of USP13 and PTEN and the decreased expression of GLUT1, HK2, 
A

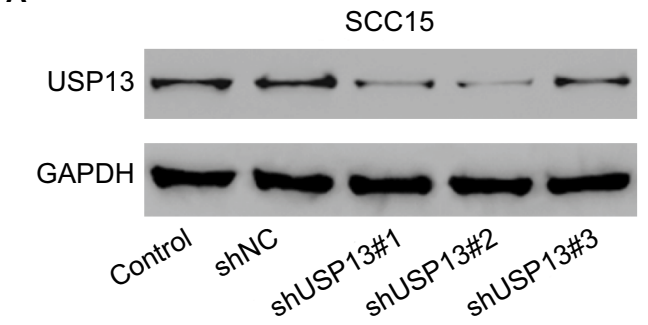

B

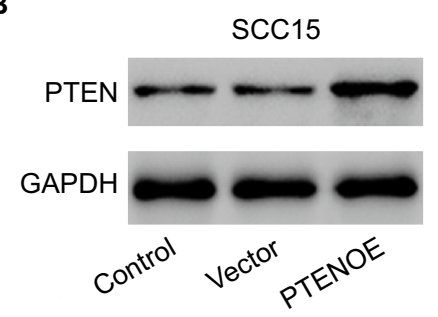

E

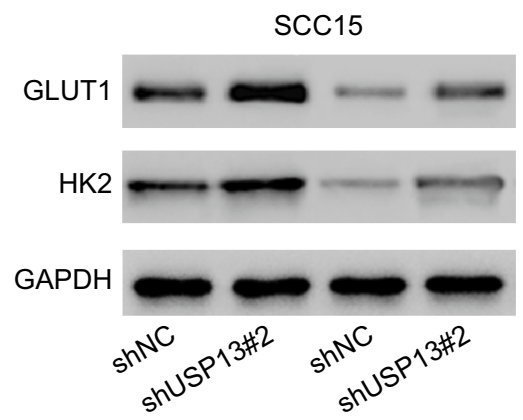

$\overline{\text { Vector }} \quad \overline{\text { PTENOE }}$
C

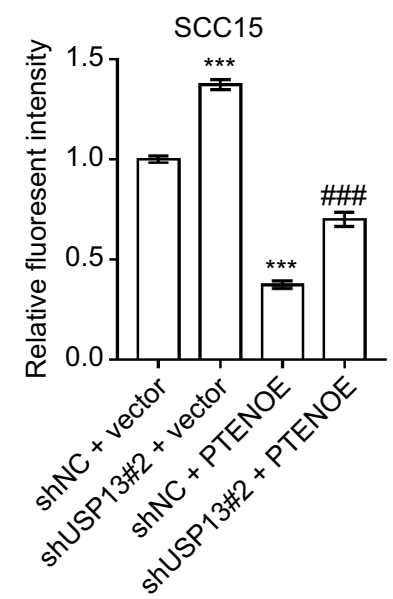

D

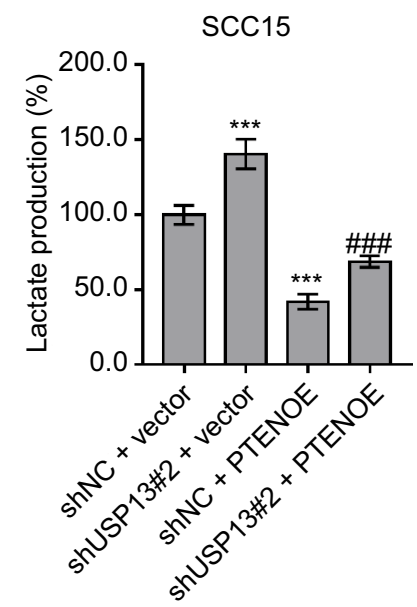

$\mathbf{F}$

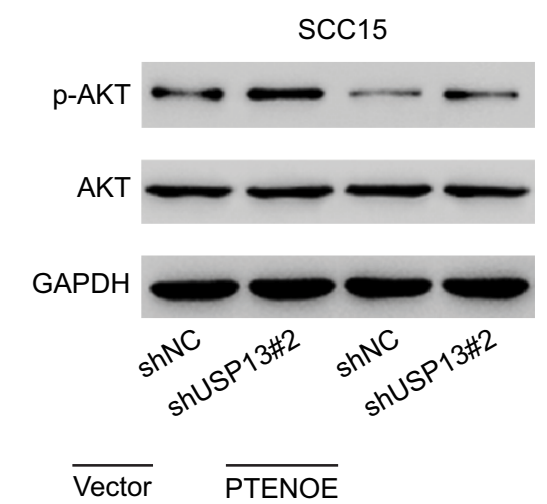

Figure 3 The PTEN/AKT pathway was involved in the biological functions of USPI 3 in OSCC cells.

Notes: (A) SCCI5 cells were transduced with USPI3 shRNAs (shUSPI3\#I, shUSPI3\#2, and shUSPI3\#3) and shNC. Cells that did not undergo any treatment served as the control. Western blot was done to evaluate the knockdown efficiency of three USPI 3 shRNAs 48 hours post treatment. (B) SCCI 5 cells were transduced with a PTENoverexpressing (PTENOE) lentivirus and a control vector virus. Western blot was carried out to detect PTEN expression 48 hours post treatment. (C-F) SCCI5 cells were transduced with shUSPI3\#2/shNC and PTENOE/vector. 2-NBDG uptake (C), lactate production (D), and expression of the related proteins were measured ( $\mathrm{n}=3$ biological replicates). ${ }^{* * *} P<0.001$ vs shNC + vector; ${ }^{\prime \prime} P<0.001$ vs shUSPI3\#2+ vector.

Abbreviations: 2-NBDG, 2-(N-[7-nitrobenz-2-oxa-I,3-diazol-4-yl]amino)-2-deoxyglucose; shNC, control shRNA; USPI3, PTEN, phosphatase and tensin homolog deleted on chromosome I0; PTENOE, PTEN-overexpressing.

and p-AKT in USP13OE xenografts, which were consistent with the results obtained in the in vitro experiments.

\section{Discussion}

Previous studies have reported the functions of USP13 in tumorigenesis. For example, USP13 stimulates melanoma cell invasion, ${ }^{20}$ drives ovarian cancer metabolism, ${ }^{21}$ and promotes the proliferation of glioma stem cells, ${ }^{22}$ which demonstrates the oncogenic roles of USP13 in these cancers. However, USP13 plays a tumor-suppressive role in breas $\mathrm{t}^{23}$ and colorectal cancer ${ }^{24}$ by regulating cell metabolism and proliferation. Here, our data first showed low expression of USP13 in OSCC tissues, which was associated with clinical stage. Ectopic expression of USP13 suppressed cell proliferation, glycolysis, and tumorigenicity. Our data together with previous reports suggest the important context-specific role of USP13 in tumorigenesis.

To explore the mechanism by which USP13 inhibited glycolysis in OSCC cells, we examined its effects on AKT signaling, which is known to be activated by USP $13 .{ }^{23}$ The AKT signaling pathway is involved in diverse biological processes, including cell proliferation and glycolysis in 
A

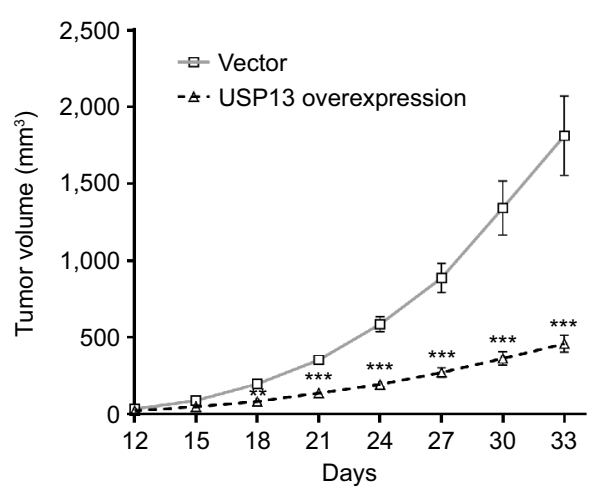

B

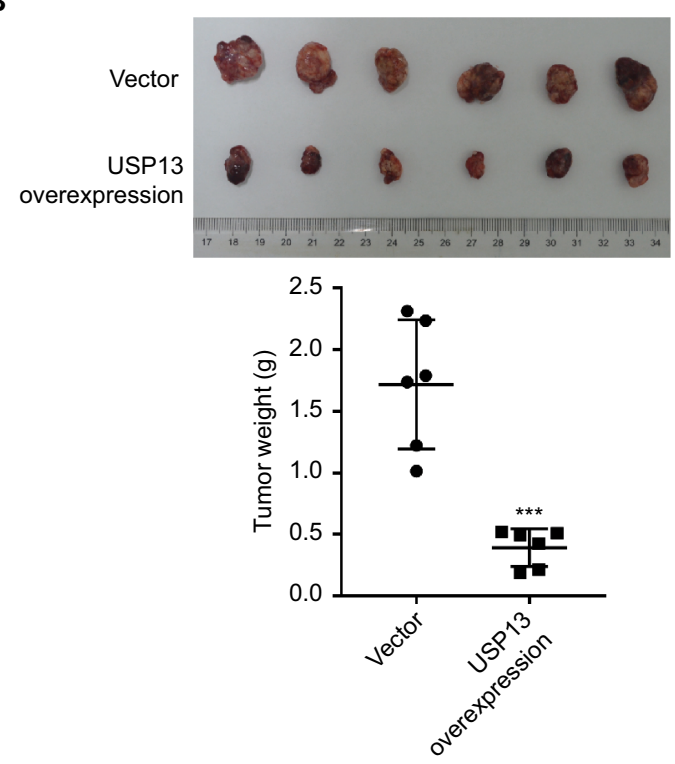

C

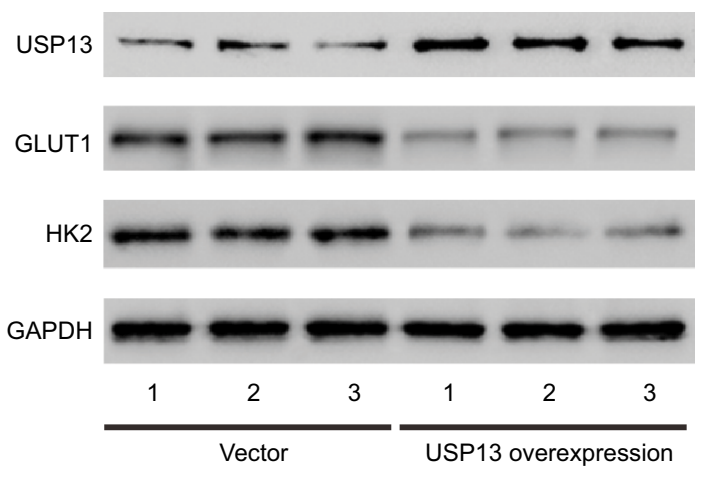

D

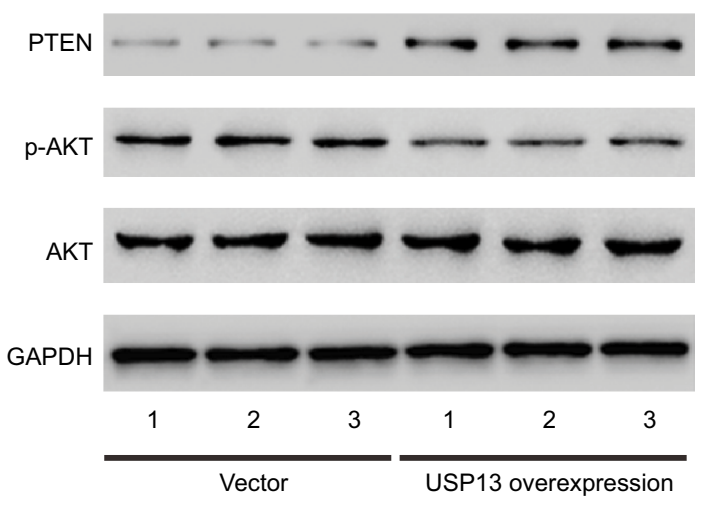

Figure 4 Effects of USPI 3 overexpression on OSCC growth in vivo.

Notes: (A) USPI 3 overexpression significantly repressed tumor growth in nude mice ( $n=6$ per group). Tumor volume was measured every 3 days. (B-D) On day 33 , after inoculation, the mice were sacrificed. The xenografts were resected and weighed (B), and partial tissues were processed for Western blotting analysis $(\mathbf{C}, \mathbf{D})$. $* * P<0.0$ I, $* * * P<0.001$ vs vector.

Abbreviations: GAPDH, glyceraldehyde-3-phosphate dehydrogenase; GLUTI, glucose transporter-I; HK2, hexokinase-2; OSCC, oral squamous cell carcinoma; PTEN, phosphatase and tensin homolog deleted on chromosome 10.

cancer cells. ${ }^{11-13}$ PTEN is a negative regulator of AKT. ${ }^{6}$ In OSCC tissues, PTEN expression is downregulated and negatively associated with activated $\mathrm{AKT} .^{7-10}$ Consistent with earlier findings, USP13 increased PTEN protein levels and decreased p-AKT levels in OSCC cells, and overexpression of PTEN partially reversed the USP13-knockdown-induced glucose uptake and lactate production in the present study.

Loss of PTEN and abnormal AKT activation influences the regulation of various downstream effectors, thus contributing to tumorigenesis. ${ }^{11-13}$ GLUT1 and HK2 are important glycolysis regulators downstream of the AKT pathway. ${ }^{26,27}$ AKT activity is essential for insulin-induced GLUT1 gene expression. ${ }^{26}$ The PI3K inhibitor LY294002 and the AKT inhibitor MK-2206 markedly decreased the protein expression of HK2 in osteosarcoma cells. ${ }^{27}$ In our experiments,
USP13 overexpression reduced the levels of GLUT1 and HK2 in OSCC cells, while knockdown of USP13 had the opposite effects. PTEN overexpression partially reversed the USP13-knockdown-induced increase in GLUT1 and HK2. Collectively, our results indicate that regulation of GLUT1 and HK2 by USP13 results from alterations in PTEN expression level and AKT activity. Accordingly, we propose that USP13 inhibits the proliferation and glycolysis of OSCC cells via the upregulation of PTEN and the downregulation of AKT activity. The effects of USP13 knockdown were not completely blocked by PTEN overexpression, which may indicate the existence of other mediators downstream USP13.

In conclusion, USP13 expression is markedly reduced in OSCC clinical samples and facilitates cell proliferation and tumorigenicity. USP13 knockdown in OSCC cells promotes 
the Warburg effect via the PTEN/AKT pathway. These findings not only help to improve our understanding of OSCC progression but also provide information for the development of novel cancer therapies.

\section{Disclosure}

The authors report no conflicts of interest in this work.

\section{References}

1. Abram MH, van Heerden WF, Rheeder P, Girdler-Brown BV, van Zyl AW. Epidemiology of oral squamous cell carcinoma. South Afr Dent J. 2012;67(10):550-553.

2. Brocklehurst PR, Baker SR, Speight PM. Oral cancer screening: what have we learnt and what is there still to achieve? Future Oncol. 2010;6(2):299-304.

3. Vander Heiden MG, Cantley LC, Thompson CB. Understanding the Warburg effect: The metabolic requirements of cell proliferation. Science. 2009;324(5930):1029-1033.

4. Koppenol WH, Bounds PL, Dang CV. Otto Warburg's contributions to current concepts of cancer metabolism. Nat Rev Cancer. 2011;11(5):325-337.

5. Bayley JP, Devilee P. The Warburg effect in 2012. Curr Opin Oncol. 2012;24(1):62-67.

6. Martini M, De Santis MC, Braccini L, Gulluni F, Hirsch E. PI3K/ Akt signaling pathway and cancer: an updated review. Ann Med. 2014;46(6):372-383.

7. Kurasawa Y, Shiiba M, Nakamura M, et al. PTEN expression and methylation status in oral squamous cell carcinoma. Oncol Rep. 2008;19(6):1429-1434.

8. Rahmani A, Alzohairy M, Babiker AY, Rizvi MA, Elkarimahmad HG. Clinicopathological significance of PTEN and Bcl2 expressions in oral squamous cell carcinoma. Int J Clin Exp Pathol. 2012;5(9):965.

9. Lim J, Kim JH, Paeng JY, et al. Prognostic value of activated Akt expression in oral squamous cell carcinoma. J Clin Pathol. 2005;58(11):1199-1205.

10. Yu Z, Weinberger PM, Sasaki C, et al. Phosphorylation of Akt (Ser473) predicts poor clinical outcome in oropharyngeal squamous cell cancer. Cancer Epidemiol Biomarkers Prev. 2007;16(3):553-558.
11. Hagiwara A, Cornu M, Cybulski N, et al. Hepatic mTORC2 activates glycolysis and lipogenesis through Akt, glucokinase, and SREBP1c. Cell Metab. 2012;15(5):725-738.

12. Elstrom RL, Bauer DE, Buzzai M, et al. Akt stimulates aerobic glycolysis in cancer cells. Cancer Res. 2004;64(11):3892-3899.

13. Vivanco I, Sawyers CL. The phosphatidylinositol 3-kinase AKT pathway in human cancer. Nat Rev Cancer. 2002;2(7):489-501.

14. Reyes-Turcu FE, Horton JR, Mullally JE, Heroux A, Cheng X, Wilkinson $\mathrm{KD}$. The ubiquitin binding domain ZNF UBP recognizes the C-terminal diglycine motif of unanchored ubiquitin. Cell. 2006;124(6):1197-1208.

15. Clague MJ, Urbé S. Ubiquitin: same molecule, different degradation pathways. Cell. 2010;143(5):682-685.

16. Chen M, Gutierrez GJ, Ronai ZA. Ubiquitin-recognition protein Ufd1 couples the endoplasmic reticulum (ER) stress response to cell cycle control. Proc Natl Acad Sci U S A. 2011;108(22):9119-9124.

17. Liu Y, Soetandyo N, Lee JG, et al. USP13 antagonizes gp78 to maintain functionality of a chaperone in ER-associated degradation. Elife. 2014;3:e01369.

18. Liu J, Xia H, Kim M, et al. Beclin1 controls the levels of $\mathrm{p} 53$ by regulating the deubiquitination activity of USP10 and USP13. Cell. 2011;147(1):223-234.

19. Sun $\mathrm{H}$, Zhang Q, Jing YY, et al. USP13 negatively regulates antiviral responses by deubiquitinating sting. Nat Commun. 2017;8:15534.

20. Zhao X, Fiske B, Kawakami A, Li J, Fisher DE. Regulation of MITF stability by the USP13 deubiquitinase. Nat Commun. 2011;2(1):414.

21. Han C, Yang L, Choi HH, et al. Amplification of USP13 drives ovarian cancer metabolism. Nat Commun. 2016;7(1):13525.

22. Fang X, Zhou W, Wu Q, et al. Deubiquitinase USP13 maintains glioblastoma stem cells by antagonizing FBXL14-mediated Myc ubiquitination. J Exp Med. 2017;214(1):245-267.

23. Zhang J, Zhang P, Wei Y, et al. Deubiquitylation and stabilization of PTEN by USP13. Nat Cell Biol. 2013;15(12):1486-1494.

24. Xiang S, Fang J, Wang S, Deng B, Zhu L. MicroRNA-135b regulates the stability of PTEN and promotes glycolysis by targeting USP13 in human colorectal cancers. Oncol Rep. 2015;33(3):1342-1348.

25. Zhou Y, Que KT, Zhang Z, et al. Iron overloaded polarizes macrophage to proinflammation phenotype through ROS/acetyl-p53 pathway. Cancer Med. 2018;7(8):4012-4022.

26. Barthel A, Okino ST, Liao J, et al. Regulation of GLUT1 gene transcription by the serine/threonine kinase Akt1.J Biol Chem. 1999;274(29):20281-20286.

27. Zhuo B, Li Y, Li Z, et al. PI3K/Akt signaling mediated hexokinase-2 expression inhibits cell apoptosis and promotes tumor growth in pediatric osteosarcoma. Biochem Biophys Res Commun. 2015;464(2):401-406. 


\section{Supplementary materials}

\section{Materials and methods}

Real-time PCR

Total RNA was extracted with Trizol reagent (Invitrogen, Carlsbad, CA, USA) following the manufacturer's protocol.
After transcribing into cDNA, real-time PCR was carried out on an ABI 7300 system (Applied Biosystem, Foster City, CA, USA) with GAPDH as interal control. The primers are listed in Table S1.

Table SI Primers for real-time PCR

\begin{tabular}{|c|c|c|}
\hline Gene & Forward primer & Reverse primer \\
\hline PKM2 & 5'-AGCAAGAAGGGTGTGAAC-3' & 5'-CGGATGAATGACGCAAAC-3' \\
\hline HIFIA & 5'-TCGGCGAAGTAAAGAATC-3' & 5'-TTCCTCACACGCAAATAG-3' \\
\hline$H K 2$ & 5'-AGGAGGATGAAGGTAGAAATGG-3' & 5'-ACTGGACAATGTGGTCAAAG-3' \\
\hline GLUTI & 5'-TGCAGGAGATGAAGGAAG-3' & 5'-CAATGGTGGCATACACAG-3' \\
\hline GLUT4 & 5'-CCCATTCCTTGGTTCATCG-3' & 5'-CCATAGCCTCCGCAACATAC-3' \\
\hline LDHA & 5'-GTTGTTGGGGTTGGTGCTG-3' & 5'-GCTTTCTCCCTCTTGCTGACG-3' \\
\hline c-Myc & 5'-ССТТСТТТССТССАСТСТС-3' & 5'-CAAACССТСТСССТTТСТС--3' \\
\hline GAPDH & 5'-AATCCCATCACCATCTTC-3' & 5'-AGGCTGTTGTCATACTTC-3' \\
\hline
\end{tabular}

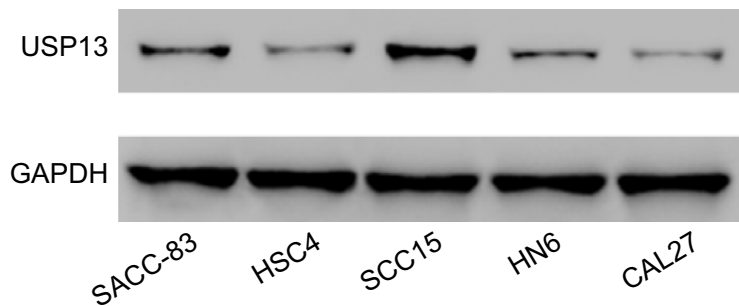

Figure SI USPI 3 expression level in five OSCC cell lines was analyzed by Western blot.

Notes: Representative blots from three independent experiments are shown All the cell lines were obtained from Cell Bank of Chinese Academy of Sciences (Shanghai, China) and cultured in high glucose DMEM (HyClone, Logan, UT, USA) supplemented with 10\% FBS (Invitrogen, Carlsbad, CA, USA) and penicillin/ streptomycin (Solarbio, Beijing, China) at $37^{\circ} \mathrm{C}$ in a moist atmosphere with $5 \% \mathrm{CO}_{2}$. Abbreviation: OSCC, oral squamous cell carcinoma.

Cancer Management and Research

\section{Publish your work in this journal}

Cancer Management and Research is an international, peer-reviewed open access journal focusing on cancer research and the optimal use of preventative and integrated treatment interventions to achieve improved outcomes, enhanced survival and quality of life for the cancer patient. The manuscript management system is completely online and includes

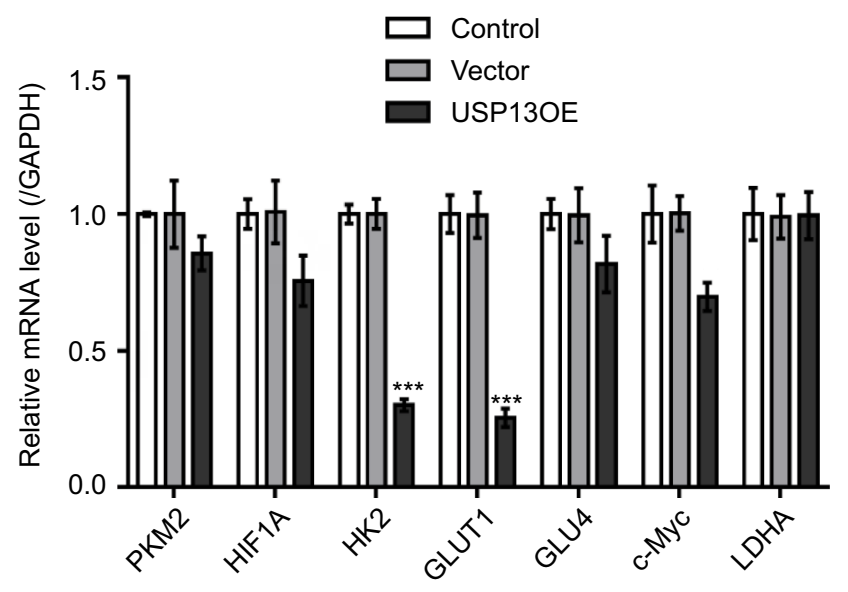

Figure S2 CAL27 cells were transduced with USPI3 overexpressing (USPI3OE) and control vector virus.

Notes: Cells without any treatment were served as control. Real-time PCR was performed to detect glycolysis-related genes at 48 hours post treatment. $* * * P<0.001$ vs Vector.

Abbreviations: GAPDH, glyceraldehyde-3-phosphate dehydrogenase; GLUT, glucose transporter-I; USPI3OE, USPI3 overexpression.

a very quick and fair peer-review system, which is all easy to use. Visit http://www.dovepress.com/testimonials.php to read real quotes from published authors. 